MATHEMATICS OF COMPUTATION

Volume 67, Number 223, July 1998, Pages 1001-1021

S 0025-5718(98)00953-3

\title{
EFFICIENT RUNGE-KUTTA INTEGRATORS FOR INDEX-2 DIFFERENTIAL ALGEBRAIC EQUATIONS
}

\author{
J. C. BUTCHER AND R. P. K. CHAN
}

\begin{abstract}
In seeking suitable Runge-Kutta methods for differential algebraic equations, we consider singly-implicit methods to which are appended diagonally-implicit stages. Methods of this type are either similar to those of Butcher and Cash or else allow for the importation of a final derivative from a previous step. For these two classes, with up to three additional diagonallyimplicit stages, we derive methods that satisfy appropriate order conditions for index-2 DAEs.
\end{abstract}

\section{INTRODUCTION}

This paper is concerned with the use of diagonal extensions to singly-implicit Runge-Kutta methods for the solution of index- 2 differential algebraic equations (DAEs). The methods we discuss fall into two main classes. The first of these is based on the use of an $n \times n$ singly-implicit block to which are appended $m$ further diagonally-implicit stages, as in Butcher and Cash [6]. In that paper, since stiff problems were the intended application, the appending of additional diagonallyimplicit stages was motivated by a wish to include all stage abscissae within the overall step, but at the same time to preserve A-stability. In the DAE context, assuming that there is no stiffness, there is no need to demand good stability in the left half-plane but only at infinity. Hence, our aim here is to add the diagonallyimplicit stages only for the purpose of improving performance of the method as a DAE solver.

In addition to standard singly-implicit methods, we consider a slight generalization originally proposed by Butcher [5]. In this generalization, the derivative of the solution used at the end of a previously completed step is used to obtain an order $n+1$ for the approximations produced in the $n \times n$ singly-implicit block with which the step commences. This is formally equivalent to regarding the method as having a first stage with zero abscissa placed before the $n \times n$ singly-implicit block. For the purposes of our search for methods for index-2 DAEs, we will add $m$ further stages after the singly-implicit block. If we interpret the method as having a preliminary explicit stage with abscissa 0 , this will make a total of $n+m+1$ stages.

In Section 2 we explore in more detail the structure of the methods we are seeking and the order conditions that will be imposed. Section 3 contains a survey

Received by the editor December 9, 1994 and, in revised form, April 16, 1997.

1991 Mathematics Subject Classification. Primary 65L05, 65L06, 65L20.

Key words and phrases. Differential algebraic systems of index 2, singly-implicit Runge-Kutta methods, diagonal extensions, Laguerre polynomials.

The first author's work was supported by the New Zealand Foundation for Research, Science and Technology. 
of properties of Laguerre and generalized Laguerre polynomials that are needed in the later parts of the paper. Section 4 reviews some known properties of singlyimplicit methods and their generalization. In Section 5 we derive the details of the extended singly-implicit methods satisfying appropriate conditions for index-2 applications. A similar analysis is carried out in Section 6, but based on generalized singly-implicit methods. Finally, in Section 7, we consider the transformations of the method coefficients necessary for efficient implementation. Those elements of the coefficient matrices, not already evaluated in Sections 5 and 6, are derived as a by-product of the use of transformations.

\section{STRUCTURE AND ORDER CONDitions}

It is widely accepted that a high stage order is a desirable property of RungeKutta methods for the numerical solution of stiff ordinary differential equation systems and differential-algebraic systems. We consider methods $(A, b, c)$ for which the stage order is $n$ and for which the step size is $w h$. Since we will be considering singly-implicit, and closely related methods, it is convenient to use the scale factor $w$ so that we will be able to normalize the repeated eigenvalue of $A$ to 1 . With this assumption, stage order $n$ will mean that $B(n)$ and $C(n)$ each holds, implying that the classical order is also at least $n$. By the condition $B(n)$ in our normalization, we mean that

$$
b^{T} P(c)=\int_{0}^{w} P(x) d x,
$$

for any polynomial $P$ of degree less than $n$, and by $C(n)$ we will mean that

$$
A P(c)=\int_{0}^{c} P(x) d x,
$$

also for any polynomial $P$ of degree less than $n$. Note that in (1) and (2) the evaluation of a polynomial with a vector argument is to be interpreted componentby-component.

In applications to index-2 DAEs of the form

$$
\begin{aligned}
y^{\prime} & =f(y, z), \\
0 & =g(y),
\end{aligned}
$$

Hairer, Lubich and Roche [9] have shown that a method having stage order $q$ with a nonsingular $A$ and a stability function $R(z)$ with $|R(\infty)|<1$, also has order $q$ in both $y$ and $z$. Furthermore, their results also enable conditions for higher order to be deduced given the stated assumptions. In particular, it is shown in [3] and [7] that to achieve order $q+1$ in $y$ requires $B(q+1)$, and order $q+1$ in $z$ requires the additional conditions

$$
\begin{aligned}
& b^{T} A^{-1} c^{q+1}=w^{q+1}, \\
& b^{T} A^{-2} c^{q+1}=(q+1) w^{q},
\end{aligned}
$$

while order $q+2$ in $y$ requires the classical order conditions, $B(q+2)$ and

$$
b^{T} A c^{q}=\frac{w^{q+2}}{(q+1)(q+2)},
$$


as well as (3) and

$$
b^{T} c A^{-1} c^{q+1}=\frac{q+1}{q+2} w^{q+2} .
$$

If $q=1$ the condition $b^{T}\left(A^{-1} c^{2}\right)^{2}=4 w^{3} / 3$ is also needed for order 3 in $y$.

Stiff accuracy is also a desirable property of Runge-Kutta methods in applications to index-2 DAEs besides stiff ordinary differential equations. Since the last stage value is the same as the update the algebraic constraint is automatically satisfied. Advantages of the property, $b^{T}=e_{s}^{T} A$, where $s$ is the number of stages, include the result for a nonsingular matrix $A$ that $b^{T} A^{-1} c^{j}=w^{j}$ for all $j=0,1,2, \ldots$ In particular, $R(\infty)=1-b^{T} A^{-1} e=0$ so that $R(z)=-b^{T} A^{-2} e / z+O\left(z^{-2}\right)$ as $z \rightarrow \infty$, and the condition (3) is automatically satisfied.

In seeking efficient methods for index-2 DAEs we develop singly-implicit blocks that have a one-point spectrum, normalized to unity for convenience, and append diagonally-implicit stages with unit diagonal entries. The extended methods we seek have the form

$$
A=\left[\begin{array}{cccccc}
a_{1,1} & \cdots & a_{1, n} & 0 & \cdots & 0 \\
\vdots & & \vdots & \vdots & & \vdots \\
a_{n, 1} & \cdots & a_{n, n} & 0 & \cdots & 0 \\
a_{n+1,1} & \cdots & a_{n+1, n} & 1 & \cdots & 0 \\
\vdots & & \vdots & \vdots & \ddots & \vdots \\
a_{n+m, 1} & \cdots & a_{n+m, n} & a_{n+m, n+1} & \cdots & 1
\end{array}\right], \quad b=\left[\begin{array}{c}
b_{1} \\
\vdots \\
b_{n} \\
b_{n+1} \\
\vdots \\
b_{n+m}
\end{array}\right], \quad c=\left[\begin{array}{c}
x_{1} \\
\vdots \\
x_{n} \\
x_{n+1} \\
\vdots \\
x_{n+m}
\end{array}\right] .
$$

The reason for appending the $m$ stages to the standard singly-implicit scheme is to improve the overall performance. Implementation costs increase with $n$ and it is hoped to obtain some of the advantages of higher order without increasing the stage order. Thus, we are looking for similar advantages in the use of diagonally extended methods for differential-algebraic equations as are claimed by Butcher and Cash [6] for stiff ordinary differential equations. We also impose stiff accuracy so that $a_{n+m, j}=b_{j}$ for $j=1, \ldots, n+m-1$, and $b_{n+m}=1$. As a useful consequence, we have $R(\infty)=0$. However, in seeking diagonal extensions of singly-implicit methods with the property of stiff accuracy and having scaled abscissae contained in the interval $[0,1]$, we would require the abscissa for the last stage to be the largest.

Similarly, we consider a generalization of the extended methods having the form

$$
\widetilde{A}=\left[\begin{array}{cc}
0 & 0 \\
a_{0} & A
\end{array}\right], \quad \widetilde{b}=\left[\begin{array}{c}
b_{0} \\
b
\end{array}\right], \quad \widetilde{c}=\left[\begin{array}{l}
0 \\
c
\end{array}\right],
$$

where $a_{0}=\left[a_{1,0}, \ldots, a_{n+m, 0}\right]^{T}$, and $A, b$ and $c$ are as prescribed along with stiff accuracy including $a_{n+m, 0}=b_{0}$. The idea is to obtain a stage order one higher than the standard method. However, an important difference is that because the matrix $\widetilde{A}$ is singular, stiff accuracy does not imply that $\widetilde{R}(\infty)=0$. In this case, $\widetilde{R}(\infty)=1-b^{T} A^{-2} c$, and $\widetilde{R}(z)=\widetilde{R}(\infty)-b^{T} A^{-3} c / z+O\left(z^{-2}\right)$ as $z \rightarrow \infty$. Thus, $\widetilde{R}(\infty)=0$ requires the additional condition $b^{T} A^{-2} c=1$.

In actual implementation, the evaluation of the derivative at the first stage would not take place because it would already have been evaluated along with the corresponding stage value as the last stage in the previous step. 
It is convenient to reformulate some of the order conditions that will be used in the subsequent analysis. With $w=\widetilde{b}^{T} \widetilde{e}$, we define

$$
\begin{gathered}
C^{\star}(q) \quad \text { if } \quad c^{k}=k A c^{k-1}, \quad k=2, \ldots, q, \\
B^{\star}(p) \quad \text { if } \quad w^{k}=k b^{T} c^{k-1}, \quad k=2, \ldots, p .
\end{gathered}
$$

It is easy to show that $C^{\star}(q)$ is equivalent to

$$
A \varphi(c)=\int_{0}^{c} \varphi(x) d x+\varphi(0)(A e-c)
$$

for any polynomial $\varphi(x)$ of degree less than $q$, and that $B^{\star}(p)$ is equivalent to

$$
b^{T} \varphi(c)=\int_{0}^{w} \varphi(x) d x+\varphi(0)\left(b^{T} e-w\right),
$$

for any polynomial $\varphi(x)$ of degree less than $p$.

Theorem 1. If $A$ is nonsingular, $C^{\star}(q)$ (respectively $C(q)$ ) is equivalent to

$$
\begin{aligned}
A^{-1} \varphi(c)= & \varphi^{\prime}(c)+\varphi^{\prime}(0)\left(A^{-1} c-e\right) \\
& +\varphi(0) A^{-1} e \quad\left(\text { respectively } \varphi^{\prime}(c)+\varphi(0) A^{-1} e\right),
\end{aligned}
$$

for any polynomial $\varphi(x)$ of degree $q$ or less.

Proof. If $C^{\star}(q)$ holds, then $c^{k}=k A c^{k-1}$ or, if $A$ is nonsingular, $A^{-1} c^{k}=k c^{k-1}$ for $k=2, \ldots, q$. Let $\varphi(x)=\sum_{k=0}^{q} d_{k} x^{k}$ so that

$$
\begin{aligned}
A^{-1} \varphi(c) & =\sum_{k=0}^{q} d_{k} A^{-1} c^{k}=\sum_{k=2}^{q} d_{k}\left(k c^{k-1}\right)+\varphi^{\prime}(0) A^{-1} c+\varphi(0) A^{-1} e \\
& =\varphi^{\prime}(c)+\varphi^{\prime}(0)\left(A^{-1} c-e\right)+\varphi(0) A^{-1} e,
\end{aligned}
$$

and the result also follows if $C(q)$ holds, for then $A^{-1} c=e$. Conversely, if $A^{-1} \varphi(c)=\varphi^{\prime}(c)+\varphi^{\prime}(0)\left(A^{-1} c-e\right)+\varphi(0) A^{-1} e$ (respectively $A^{-1} \varphi(c)=\varphi^{\prime}(c)+$ $\left.\varphi(0) A^{-1} e\right)$ holds for a polynomial $\varphi(x)$ of degree $q$ or less, then $\varphi(c)=A \varphi^{\prime}(c)+$ $\varphi^{\prime}(0)(c-A e)+\varphi(0) e$ (respectively $\left.\varphi(c)=A \varphi^{\prime}(c)+\varphi(0) e\right)$ holds in particular for $\varphi(x)=x^{k}, k=1, \ldots, q$. This yields $C^{\star}(q)$ (respectively $C(q)$ ).

Corollary 1. Let $C^{\star}(q)$ (respectively $C(q)$ ) hold with stiff accuracy. If $A$ is nonsingular and $\varphi(x)$ denotes a polynomial of degree $q$ or less, then

$$
\begin{aligned}
b^{T} A^{-2} \varphi(c)= & \varphi^{\prime}(w)-\varphi^{\prime}(0) \widetilde{R}(\infty) \\
& +\varphi(0) b^{T} A^{-2} e \quad\left(\text { respectively } \quad \varphi^{\prime}(w)+\varphi(0) b^{T} A^{-2} e\right),
\end{aligned}
$$

and, if in addition $B^{\star}(q+1)$ (respectively $\left.B(q+1)\right)$ holds, then

$$
\begin{array}{r}
b^{T} c A^{-1} \varphi(c)=w \varphi(w)- \\
\int_{0}^{w} \varphi(x) d x+\varphi^{\prime}(0)\left(b^{T} c A^{-1} c-w^{2} / 2\right)+\varphi(0) b^{T} c A^{-1} e \\
\left(\text { respectively }=w \varphi(w)-\int_{0}^{w} \varphi(x) d x+\varphi(0) b^{T} c A^{-1} e\right) .
\end{array}
$$

Proof. Since $C^{\star}(q)$ holds, it follows from Theorem 1 that

$$
\begin{aligned}
A^{-2} \varphi(c) & =A^{-1} \varphi^{\prime}(c)+\varphi^{\prime}(0)\left(A^{-2} c-A^{-1} e\right)+\varphi(0) A^{-2} e, \\
b^{T} A^{-2} \varphi(c) & =b^{T} A^{-1} \varphi^{\prime}(c)+\varphi^{\prime}(0)\left(b^{T} A^{-2} c-b^{T} A^{-1} e\right)+\varphi(0) b^{T} A^{-2} e \\
& =\varphi^{\prime}(w)-\varphi^{\prime}(0) \widetilde{R}(\infty)+\varphi(0) b^{T} A^{-2} e .
\end{aligned}
$$


The result for $C(q)$ follows since it is equivalent to $C^{\star}(q)$ and $C(1)$ with the latter implying that $\widetilde{R}(\infty)=0$. If $B^{\star}(q+1)$ holds in addition to $C^{\star}(q)$, then Theorem 1 and (9) yield

$$
\begin{aligned}
b^{T} c A^{-1} \varphi(c) & =b^{T} c \varphi^{\prime}(c)+\varphi^{\prime}(0)\left(b^{T} c A^{-1} c-b^{T} c\right)+\varphi(0) b^{T} c A^{-1} e \\
& =\int_{0}^{w} x \varphi^{\prime}(x) d x+\varphi^{\prime}(0)\left(b^{T} c A^{-1} c-\frac{1}{2} w^{2}\right)+\varphi(0) b^{T} c A^{-1} e \\
& =w \varphi(w)-\int_{0}^{w} \varphi(x) d x+\varphi^{\prime}(0)\left(b^{T} c A^{-1} c-\frac{1}{2} w^{2}\right)+\varphi(0) b^{T} c A^{-1} e .
\end{aligned}
$$

If $B(q+1)$ holds in addition to $C(q)$, then $A^{-1} c=e$ and the coefficient of $\varphi^{\prime}(0)$ vanishes giving the result in this case.

In this paper we seek stiffly accurate extended methods (respectively their generalizations) for the cases $m=1,2,3$ having stage order $n$ (respectively $n+1$ ) with order $n+[(m+1) / 2]$ (respectively $n+m)$ in $y$ and order $n+[m / 2]$ (respectively $n+1+[m / 2])$ in $z$ for index-2 DAE applications.

The analysis involves substantial use of the properties of Laguerre polynomials. These properties as well as those of the generalized Laguerre polynomials are summarized in the next section for convenience of later reference. The details are given in the sections that follow.

\section{REVIEW OF LAGUERRE POLYNOMIALS}

In this section, we survey some properties of the Laguerre polynomials $L_{n}$ and the generalized Laguerre polynomials $L_{n}^{(1)}$. In each case these polynomials are defined with degrees $n=0,1,2, \ldots$, by the orthogonality conditions

$$
\int_{0}^{\infty} L_{m}(x) L_{n}(x) \exp (-x) d x=\int_{0}^{\infty} L_{m}^{(1)}(x) L_{n}^{(1)}(x) x \exp (-x) d x=0, \quad m \neq n,
$$

and by the normalizations

$$
L_{n}(0)=1, \quad L_{n}^{(1)}(0)=n+1 .
$$

All the results are standard (see, for example, [1] or [8]) or are easily derivable from standard results. They are presented here for easy reference in the later sections of the paper. They are organized for convenience as collections of closely related formulas in the form of theorems and corollaries stated without proof.

Theorem 2. The polynomials $L_{n}$ and $L_{n}^{(1)}$ are given by

$$
\begin{aligned}
L_{n}(x) & =\sum_{i=0}^{n}\left(\begin{array}{c}
n \\
i
\end{array}\right) \frac{(-x)^{i}}{i !}, \\
L_{n}^{(1)}(x) & =\sum_{i=0}^{n}\left(\begin{array}{c}
n+1 \\
i+1
\end{array}\right) \frac{(-x)^{i}}{i !} .
\end{aligned}
$$

Corollary 2. The squared norms of $L_{n}$ and $L_{n}^{(1)}$ are given by

$$
\begin{gathered}
\int_{0}^{\infty} L_{n}(x) L_{n}(x) \exp (-x) d x=1, \\
\int_{0}^{\infty} L_{n}^{(1)}(x) L_{n}^{(1)}(x) x \exp (-x) d x=n+1 .
\end{gathered}
$$




\section{Theorem 3.}

$$
\begin{aligned}
& n L_{n}(x)+(x-2 n+1) L_{n-1}(x)+(n-1) L_{n-2}(x)=0, \quad n=2,3, \ldots, \\
& n L_{n}^{(1)}(x)+(x-2 n) L_{n-1}^{(1)}(x)+n L_{n-2}^{(1)}(x)=0, \quad n=2,3, \ldots, \\
& x L_{n}^{\prime}(x)-n\left(L_{n}(x)-L_{n-1}(x)\right)=0, \quad n=1,2, \ldots, \\
& x L_{n}^{(1) \prime}(x)-n L_{n}^{(1)}(x)+(n+1) L_{n-1}^{(1)}(x)=0, \quad n=1,2, \ldots, \\
& L_{n}^{\prime}(x)-L_{n-1}^{\prime}(x)+L_{n-1}(x)=0, \quad n=1,2, \ldots, \\
& L_{n}^{(1) \prime}(x)-L_{n-1}^{(1)}{ }^{\prime}(x)+L_{n-1}^{(1)}(x)=0, \quad n=1,2, \ldots
\end{aligned}
$$

\section{Theorem 4.}

$$
\begin{aligned}
\int_{0}^{x} L_{n}(t) d t & =L_{n}(x)-L_{n+1}(x) \\
\int_{0}^{x} L_{n}^{(1)}(t) d t & =1+L_{n}^{(1)}(x)-L_{n+1}^{(1)}(x) \\
\int_{0}^{x} t L_{n}(t) d t & =(x-1) L_{n}(x)+(2-x) L_{n+1}(x)-L_{n+2}(x) \\
\int_{0}^{x} t L_{n}^{(1)}(t) d t & =(x-1) L_{n}^{(1)}(x)+(2-x) L_{n+1}^{(1)}(x)-L_{n+2}^{(1)}(x), \\
\int_{0}^{x} t^{2} L_{n}^{(1)}(t) d t= & \left(x^{2}-2 x+2\right) L_{n}^{(1)}(x)+\left(-x^{2}+4 x-6\right) L_{n+1}^{(1)}(x) \\
& +(-2 x+6) L_{n+2}^{(1)}(x)-2 L_{n+3}^{(1)}(x) .
\end{aligned}
$$

Theorem 5. For $n=1,2, \ldots$,

$$
\begin{aligned}
(y-x) \sum_{k=0}^{n-1} L_{k}(x) L_{k}(y) & =n\left(L_{n}(x) L_{n-1}(y)-L_{n}(y) L_{n-1}(x)\right) \\
(y-x) \sum_{k=0}^{n-1} \frac{L_{k}^{(1)}(x) L_{k}^{(1)}(y)}{k+1} & =L_{n}^{(1)}(x) L_{n-1}^{(1)}(y)-L_{n}^{(1)}(y) L_{n-1}^{(1)}(x) .
\end{aligned}
$$

Corollary 3. Let $x_{1}, x_{2}, \ldots, x_{n}$ denote the zeros of $L_{n}$ (respectively $\left.L_{n}^{(1)}\right)$. The inverse of the matrix with $(i, j)$-th element given by $L_{j-1}\left(x_{i}\right)$ (respectively $\left.x_{i} L_{j-1}^{(1)}\left(x_{i}\right) / j\right)$ is the matrix with $(i, j)$-th element given by $x_{j} L_{i-1}\left(x_{j}\right) / n^{2} L_{n-1}\left(x_{j}\right)^{2}$ (respectively $\left.L_{i-1}^{(1)}\left(x_{j}\right) /(n+1) L_{n-1}^{(1)}\left(x_{j}\right)^{2}\right)$.

\section{Single IMPLiCitness GENERALIZED}

Consider an $n$-stage Runge-Kutta method,

$$
A=\left[\begin{array}{ccc}
a_{1,1} & \cdots & a_{1, n} \\
\vdots & & \vdots \\
a_{n, 1} & \cdots & a_{n, n}
\end{array}\right], \quad b=\left[\begin{array}{c}
b_{1} \\
\vdots \\
b_{n}
\end{array}\right], \quad c=\left[\begin{array}{c}
x_{1} \\
\vdots \\
x_{n}
\end{array}\right] .
$$

If the stage order is $n$ and the abscissae distinct, the matrix $A$ is determined by $C(n)$ and the vector of weights $b$ by $B(n)$ in terms of $w=b^{T} e$. For if $V=\left[e, c, \ldots, c^{n-1}\right]$ 
and $C=\operatorname{diag}\left(x_{1}, \ldots, x_{n}\right)$, then

$$
\begin{aligned}
A V & =\left[c, \frac{1}{2} c^{2}, \ldots, \frac{1}{n} c^{n}\right]=C V \operatorname{diag}\left(1, \frac{1}{2}, \ldots, \frac{1}{n}\right), \\
b^{T} V & =\left[w, \frac{1}{2} w^{2}, \ldots, \frac{1}{n} w^{n}\right],
\end{aligned}
$$

and the nonsingularity of $A$ follows from that of the Vandermonde matrix $V$.

The method will be generalized by importing to the current step the derivatives computed in the previous step. This is equivalent to the Runge-Kutta matrix having a first row of zeros. The generalized method $(\widetilde{A}, \widetilde{b}, \widetilde{c})$ thus formally consists of $n+1$ stages and is partitioned according to (7), where $a_{0}=\left[a_{1,0}, \ldots, a_{n, 0}\right]^{T}$. It is constructed slightly differently to give a stage order one higher than the standard method but implementable at little additional cost. With stage order $n+1$, the matrix $A$ is now determined by $C^{\star}(n+1)$, the vector $a_{0}$ by $C(1)$, the weight vector $b$ by $B^{\star}(n+1)$, and $b_{0}$ by $B(1)$, for

$$
\begin{aligned}
A C V & =\left[\frac{1}{2} c^{2}, \frac{1}{3} c^{3}, \ldots, \frac{1}{n+1} c^{n+1}\right]=C^{2} V \operatorname{diag}\left(\frac{1}{2}, \frac{1}{3}, \ldots, \frac{1}{n+1}\right), \\
a_{0} & =c-A e \\
b^{T} C V & =\left[\frac{1}{2} w^{2}, \frac{1}{3} w^{3}, \ldots, \frac{1}{n+1} w^{n+1}\right], \\
b_{0} & =w-b^{T} e,
\end{aligned}
$$

and $A$ will be nonsingular if the components of $c$ are distinct.

If $A$ has a one-point spectrum, the singly-implicit (respectively generalized singlyimplicit) method will be completely determined in terms of a single parameter $w=b^{T} e$ (respectively $w=\widetilde{b}^{T} \widetilde{e}$ ) as a consequence of the following theorem.

Theorem 6. Let the matrix $A$ have an $n$-fold eigenvalue 1. If $C(n)$ (respectively $C^{\star}(n+1)$ ) holds, then the abscissae $x_{1}, \ldots, x_{n}$ are the zeros of $L_{n}(x)$ (respectively $\left.L_{n}^{(1)}(x)\right)$.

Proof. If $C(n)$ holds, then $(I-A) L_{k}(c)=L_{k}(c)-\int_{0}^{c} L_{k}(x) d x=L_{k+1}(c)$ for all $k<n$, and hence $L_{n}(c)=(I-A)^{n} L_{0}(c)=0$. If $C^{\star}(n+1)$ holds, then by (8), Theorems 4 and 3 , we have

$$
\begin{aligned}
(I-A)\left(c L_{k}^{(1)}(c)\right) & =c L_{k}^{(1)}(c)-\int_{0}^{c} x L_{k}^{(1)}(x) d x \\
& =L_{k}^{(1)}(c)+(c-2 e) L_{k+1}^{(1)}(c)+L_{k+2}^{(1)}(c) \\
& =\frac{k+1}{k+2} c L_{k+1}^{(1)}(c), \quad k<n, \\
\frac{c L_{n}^{(1)}(c)}{n+1} & =(I-A)\left(\frac{c L_{n-1}^{(1)}(c)}{n}\right)=\cdots=(I-A)^{n}\left(c L_{0}^{(1)}(c)\right)=0 .
\end{aligned}
$$

Let the $n$ zeros of $L_{n}(x)$ (respectively $L_{n}^{(1)}(x)$ ) be ordered according to $x_{1}<$ $x_{2}<\cdots<x_{n}$. If the choice $w=x_{n}$ is made we will have stiff accuracy for then $b^{T}=e_{n}^{T} A$ (respectively $b^{T}=e_{n}^{T} A$ and $b_{0}=e_{n}^{T} a_{0}$ ). Furthermore, the eigenvalue when scaled is given by $1 / x_{n}$ and all the abscissae will be contained in the interval $[0,1]$. In the case of the singly-implicit method, $R(\infty)=1-b^{T} A^{-1} e=0$ follows and the method is unique with stage order $n$, and order $n$ in both $y$ and $z$ for index-2 DAEs. The $(n+1)$-stage stiffly accurate generalized method is also unique with stage order $n+1$ and will have order $n+1$ in both $y$ and $z$ for index-2 DAEs 
provided that $|\widetilde{R}(\infty)|<1$. However, $\widetilde{R}(\infty)=1-b^{T} A^{-2} c$ is determined as in the following theorem.

Theorem 7. For the stiffly accurate generalized singly-implicit method $(\widetilde{A}, \widetilde{b}, \widetilde{c})$ with $n+1$ stages and stage order $n+1, \widetilde{R}(\infty)=L_{n}(w)$.

Proof. Since $C^{\star}(n+1)$ holds, application of Corollary 1 gives

$$
b^{T} A^{-2}\left(c L_{n}^{(1)}(c)\right)=\left(w L_{n}^{(1)}(w)\right)^{\prime}-(n+1) \widetilde{R}(\infty)
$$

Since $L_{n}^{(1)}(c)=0$ by Theorem 6 , we have $b^{T} A^{-2}\left(c L_{n}^{(1)}(c)\right)=0$ and $\left(w L_{n}^{(1)}(w)\right)^{\prime}=$ $w L_{n}^{(1)^{\prime}}(w)$, and the result follows by applying the appropriate identities in Theorem 3 .

Numerical computations show that $L_{n}(w)$ is not bounded by 1 when $w$ is the largest zero of $L_{n}^{(1)}(x)$. Thus the stiffly accurate generalized method with $n+1$ stages cannot attain order $n+1$ in either $y$ or $z$ for index-2 DAEs with all of its scaled abscissae contained in the interval $[0,1]$. This provides a motivation for appending one or more diagonally-implicit stages to the generalized method.

Theorem 8. For the singly-implicit method $(A, b, c)$ with $n$ stages and stage order $n, B(n+1)$ holds if and only if $L_{n}^{(1)}(w)=0$.

Proof. Since $B(n)$ holds, applying (1) with $P(x)=L_{n}(x)-(-1)^{n} x^{n} / n$ ! yields

$$
b^{T} L_{n}(c)=\int_{0}^{w} L_{n}(x) d x+\frac{(-1)^{n}}{n !}\left(b^{T} c^{n}-\frac{w^{n+1}}{n+1}\right) .
$$

Now since $L_{n}(c)=0$ and $\int_{0}^{w} L_{n}(x) d x=L_{n}(w)-L_{n+1}(w)=w L_{n}^{(1)}(w) /(n+1)$, the result follows as $w \neq 0$.

$B(n+1)$ does not hold if stiff accuracy is imposed because $L_{n}^{(1)}(x) \neq 0$ for any zero of $L_{n}(x)$. Hence order $n+1$ in $y$ cannot be attained by such a stiffly accurate method. Without stiff accuracy an order $n+1$ in $y$ and order $n$ in $z$ can be attained if $|R(\infty)|<1$ for $w$ satisfying $L_{n}^{(1)}(w)=0$. By Theorems 1 and 6 , we have $0=b^{T} A^{-1} L_{n}(c)=L_{n}(w)-R(\infty)$ and $w$ would need to be chosen so that $\left|L_{n}(w)\right|<1$.

It is natural to require that all the abscissae fall within the step. For both the singly-implicit method and its generalization this conflicts with the conditions for higher order, good stability behaviour at infinity and stiff accuracy. Hence, we consider the addition of further diagonally-implicit stages in the following sections.

\section{EXTENDED SINGLY-IMPLICIT METHODS}

We consider the construction of an $(n+m)$-stage singly-implicit method by appending $m$ diagonally-implicit stages to the $n$ singly-implicit stages and impose stiff accuracy for the cases $m=1,2,3$. In this section we will always assume a stage order of $n,(I-A)^{n+m}=0, b^{T}=e_{n+m}^{T} A$ and $R(\infty)=0$. 
5.1. One appended diagonally-implicit stage. For $m=1$, we have

$$
A=\left[\begin{array}{cccc}
a_{1,1} & \cdots & a_{1, n} & 0 \\
\vdots & & \vdots & \vdots \\
a_{n, 1} & \cdots & a_{n, n} & 0 \\
b_{1} & \cdots & b_{n} & 1
\end{array}\right], \quad b=\left[\begin{array}{c}
b_{1} \\
\vdots \\
b_{n} \\
1
\end{array}\right], \quad c=\left[\begin{array}{c}
x_{1} \\
\vdots \\
x_{n} \\
w
\end{array}\right] .
$$

As in Section 4, the positive abscissae $x_{1}<\cdots<x_{n}$ are the distinct zeros of $L_{n}(x)$, the $a_{i, j}$ are determined by $C(n)$, and the weight vector $b$ by $B(n)$.

Theorem 9. $B(n+1)$ holds if and only if $L_{n+1}(w)=0$.

Proof. By $B(n)$, applying (1) with $P(x)=L_{n}(x)-(-1)^{n} x^{n} / n$ ! yields

$$
b^{T} L_{n}(c)=\int_{0}^{w} L_{n}(x) d x+\frac{(-1)^{n}}{n !}\left(b^{T} c^{n}-\frac{w^{n+1}}{n+1}\right) .
$$

Since $b^{T} L_{n}(c)=L_{n}(w)$ and $\int_{0}^{w} L_{n}(x) d x=L_{n}(w)-L_{n+1}(w)$, we have

$$
L_{n+1}(w)=\frac{(-1)^{n}}{n !}\left(b^{T} c^{n}-\frac{w^{n+1}}{n+1}\right),
$$

and hence the result.

If $B(n+1)$ holds we have a method of order $n+1$ in $y$ and order $n$ in $z$ for index-2 DAEs. By the interlacing property of the zeros of Laguerre polynomials, the largest is the only zero of $L_{n+1}(x)$ greater than $x_{n}$. Choosing $w$ as this largest zero results in all the scaled abscissae lying in $[0,1]$.

5.2. Two appended diagonally-implicit stages. In this subsection we consider the case $m=2$ consisting of $n$ singly-implicit stages with two appended diagonallyimplicit stages,

$$
A=\left[\begin{array}{ccccc}
a_{1,1} & \cdots & a_{1, n} & 0 & 0 \\
\vdots & & \vdots & \vdots & \vdots \\
a_{n, 1} & \cdots & a_{n, n} & 0 & 0 \\
a_{n+1,1} & \cdots & a_{n+1, n} & 1 & 0 \\
b_{1} & \cdots & b_{n} & b_{n+1} & 1
\end{array}\right], \quad b=\left[\begin{array}{c}
b_{1} \\
\vdots \\
b_{n} \\
b_{n+1} \\
1
\end{array}\right], \quad c=\left[\begin{array}{c}
x_{1} \\
\vdots \\
x_{n} \\
v \\
w
\end{array}\right] .
$$

In addition to stiff accuracy, stage order $n$ and $(I-A)^{n+2}=0$, we will impose the conditions $B(n+1)$ and $b^{T} A^{-2} c^{n+1}=(n+1) w^{n}$ to obtain order $n+1$ in both $y$ and $z$ with $w$ as parameter and consider some choices of $w$.

\section{Theorem 10.}

$$
v=n+1-\frac{n L_{n}(w)}{L_{n+1}(w)} .
$$

Proof. By (1), $B(n+1)$ implies

$$
\begin{aligned}
b_{n+1} L_{n}(v)+L_{n}(w) & =b^{T} L_{n}(c)=\int_{0}^{w} L_{n}(x) d x=L_{n}(w)-L_{n+1}(w), \\
b_{n+1} L_{n}(v) & =-L_{n+1}(w) .
\end{aligned}
$$


Applying Corollary 1 with $\varphi(x)=x L_{n}(x)-(-1)^{n} x^{n+1} / n !$, and $b^{T} A^{-2} c^{n+1}=$ $(n+1) w^{n}$, we obtain

$$
\begin{aligned}
-b_{n+1} v L_{n}(v)+w L_{n}(w) & =b^{T} A^{-2} c L_{n}(c)=\left(w L_{n}(w)\right)^{\prime}=w L_{n}^{\prime}(w)+L_{n}(w), \\
b_{n+1} v L_{n}(v) & =n L_{n}(w)-(n+1) L_{n+1}(w) .
\end{aligned}
$$

Hence $(v-n-1) L_{n+1}(w)=-n L_{n}(w)$. Since $L_{n+1}(w) \neq 0$, for otherwise $L_{n}(w)=0$ results in a contradiction, the desired result follows.

We remark that once $w$ and $v$ are known $b_{n+1}$ can be determined from (25).

The choice $w=x_{n}$ leads to $v=n+1$ and, except for the case $n=1, w>v$ so that all the abscissae are contained in the interval $[0,1]$ when scaled by the factor $1 / w$.

The next theorem gives some other possible choices of the parameter $w$.

\section{Theorem 11.}

$$
\begin{aligned}
& \text { (a) } b^{T} A c^{n}=\frac{w^{n+2}}{(n+1)(n+2)} \text { if and only if } L_{n+2}(w)=0 ; \\
& \text { (b) } B(n+2) \text { if and only if }(n+1) L_{n+2}(w)=n L_{n+1}(w) ; \\
& \text { (c) } b^{T} A^{-2} e=0 \text { if and only if } L_{n+1}^{(1)}(w)=0 .
\end{aligned}
$$

Proof. (a) By $C(n)$ and $B(n+1)$, applying (1) and (2) with $P(x)=L_{n}(x)-$ $(-1)^{n} x^{n} / n$ ! gives

$$
b^{T} A L_{n}(c)=\int_{0}^{w}\left(\int_{0}^{x} L_{n}(t) d t\right) d x+\frac{(-1)^{n}}{n !}\left(b^{T} A c^{n}-\frac{w^{n+2}}{(n+1)(n+2)}\right) .
$$

Since $b^{T} A L_{n}(c)=2 b_{n+1} L_{n}(v)+L_{n}(w)$, and the integral evaluates to $L_{n}(w)-$ $2 L_{n+1}(w)+L_{n+2}(w)$, use of $(25)$ then gives

$$
\frac{(-1)^{n}}{n !}\left(b^{T} A c^{n}-\frac{w^{n+2}}{(n+1)(n+2)}\right)=-L_{n+2}(w)
$$

and the result follows.

(b) By $B(n+1)$, applying (1) with $P(x)=x L_{n}(x)-(-1)^{n} x^{n+1} / n$ ! gives

$$
b^{T} c L_{n}(c)=\int_{0}^{w} x L_{n}(x) d x+\frac{(-1)^{n}}{n !}\left(b^{T} c^{n+1}-\frac{w^{n+2}}{n+2}\right) .
$$

Since $b^{T} c L_{n}(c)=b_{n+1} v L_{n}(v)+w L_{n}(w)$, and the integral gives $(w-1) L_{n}(w)+$ $(2-w) L_{n+1}(w)-L_{n+2}(w)$ by Theorem 4 , we have by the use of (26) and Theorem 3 ,

$$
\frac{(-1)^{n}}{n !}\left(b^{T} c^{n+1}-\frac{w^{n+2}}{n+2}\right)=n L_{n+1}(w)-(n+1) L_{n+2}(w) .
$$

The result then follows.

(c) By $C(n)$, applying Corollary 1 with $\varphi(x)=L_{n}(x)$ gives

$$
b^{T} A^{-2} L_{n}(c)=L_{n}^{\prime}(w)+b^{T} A^{-2} e .
$$

Since $b^{T} A^{-2} L_{n}(c)=L_{n}(w)-b_{n+1} L_{n}(v)=L_{n}(w)+L_{n+1}(w)=L_{n+1}^{(1)}(w)-L_{n-1}^{(1)}(w)$ $=L_{n+1}^{(1)}(w)+L_{n}^{\prime}(w)$ by $(25)$ and Theorem 3 , the result follows. 
It is desirable that $w$ be chosen as the largest of the abscissae so that when scaled by the factor $1 / w$, all the abscissae are contained in the interval $[0,1]$. The theorem allows for such a choice of $w$ with $w>v$ and $w>x_{n}$, where $x_{n}$ is the largest zero of $L_{n}(x)$.

If $L_{n+2}(w)=0$, then Theorem 3 gives $(n+1) L_{n}(w)=(2 n+3-w) L_{n+1}(w)$ and, by Theorem 10, we have $v=\left(n w-n^{2}-n+1\right) /(n+1)$. Hence $w-v=$ $n+1+(v-1) / n=n+(w-1) /(n+1)$. If $w$ is the largest zero of $L_{n+2}(x)$, then $w>n+2$, the average of the zeros. Hence $w>v>0$. It is also clear that $w>x_{n}$.

If $(n+1) L_{n+2}(w)=n L_{n+1}(w)$ and $L_{n+2}(w)$ is expressed in terms of $L_{n}(w)$ and $L_{n+1}(w)$, Theorem 10 yields $v=(n(n+1) w+1) /(n+1)^{2}$. Hence $w-v=$ $(w-1 /(n+1)) /(n+1)=(v-1 /(n+1)) / n$. The polynomial $(n+1) L_{n+2}(x)-$ $n L_{n+1}(x)$ has alternating signs and $n+2$ positive zeros with average $2+n^{2} /$ $(n+1)$. Thus there exist values of $w$ greater than $v$ and if $w$ is the largest zero, then numerical computations show that $w>x_{n}$.

If $b^{T} A^{-2} e=0$ which implies $R(z)=O\left(z^{-2}\right)$ as $z \rightarrow \infty$, then $L_{n+1}^{(1)}(w)=0$. Hence $L_{n+1}(w)=L_{n+2}(w)$ and, when $L_{n+2}(w)$ is expressed in terms of $L_{n}(w)$ and $L_{n+1}(w)$, Theorem 10 then yields $v=1+n w /(n+1)$. Hence $w-v=$ $(w-n-1) /(n+1)=(v-n-1) / n$. Now if $w$ is the largest zero of $L_{n+1}^{(1)}(x)$, then $w>n+2$, the average of the zeros, so that $w>v>n+1$. It is also true that $w>x_{n}$.

Finally, we remark that the condition $b^{T}\left(c^{n+1}-(n+1) A c^{n}\right)=0$ cannot hold because it can be shown to imply $L_{n+1}(w)=0$ which gives a contradiction as $L_{n}(w)=0$ is also implied.

5.3. Three appended diagonally-implicit stages. We now consider the case $m=3$ consisting of $n$ singly-implicit stages with three appended diagonally implicit stages,

$$
A=\left[\begin{array}{cccccc}
a_{1,1} & \cdots & a_{1, n} & 0 & 0 & 0 \\
\vdots & & \vdots & \vdots & \vdots & \vdots \\
a_{n, 1} & \cdots & a_{n, n} & 0 & 0 & 0 \\
a_{n+1,1} & \cdots & a_{n+1, n} & 1 & 0 & 0 \\
a_{n+2,1} & \cdots & a_{n+2, n} & a_{n+2, n+1} & 1 & 0 \\
b_{1} & \cdots & b_{n} & b_{n+1} & b_{n+2} & 1
\end{array}\right], \quad b=\left[\begin{array}{c}
b_{1} \\
\vdots \\
b_{n} \\
b_{n+1} \\
b_{n+2} \\
1
\end{array}\right], \quad c=\left[\begin{array}{c}
x_{1} \\
\vdots \\
x_{n} \\
u \\
v \\
w
\end{array}\right]
$$

In addition to stiff accuracy, stage order $n$ and $(I-A)^{n+3}=0$, we will impose $B(n+2)$ and some additional conditions to obtain order $n+2$ in $y$ and order $n+1$ in $z$ in terms of $w=b^{T} e$. A condition for order $n+1$ in $z$ given by (4) and conditions for order $n+2$ in $y$ by (5) and (6).

Theorem 12. $b^{T} A^{-2} e=L_{n+2}^{(1)}(w)$.

Proof. $A^{-1}=\sum_{i=0}^{n+2}\left(\begin{array}{c}n+3 \\ i+1\end{array}\right)(-1)^{i} A^{i}$. Pre-multiplying by $b^{T} A^{-1}$ and post-multiplying by $e$, the result follows from the conditions $C(n), B(n+1)$ and $(5)$.

In the remainder of this section we consider the determination of $u, v, w, b_{n+1}$, $b_{n+2}$ and $a_{n+2, n+1}$. By $B(n+2)$, we have

$$
b_{n+2}(v-u) L_{n}(v)+(w-u) L_{n}(w)=b^{T}(c-u e) L_{n}(c)=\int_{0}^{w}(x-u) L_{n}(x) d x .
$$


Applying Corollary 1 with $\varphi(x)=(x-u) L_{n}(x)-(-1)^{n} x^{n+1} / n !, C(n),(4)$ and Theorem 12 yield

$$
\begin{aligned}
-b_{n+2}(v-u) L_{n}(v)+(w-u) L_{n}(w) & =b^{T} A^{-2}(c-u e) L_{n}(c) \\
& =(w-u) L_{n}^{\prime}(w)+L_{n}(w)-u L_{n+2}^{(1)}(w) .
\end{aligned}
$$

Theorem 13.

$$
u=n+1-n \frac{L_{n+1}(w)}{L_{n+2}(w)} .
$$

Proof. By adding (27) and (28) we obtain an explicit expression for $u$,

$$
u=\frac{w L_{n}^{\prime}(w)+(1-2 w) L_{n}(w)+\int_{0}^{w} x L_{n}(x) d x}{L_{n+2}^{(1)}(w)+L_{n}^{\prime}(w)-2 L_{n}(w)+\int_{0}^{w} L_{n}(x) d x} .
$$

The result follows by use of the identities in Theorems 3 and 4 .

Similarly, by considering the polynomial $(x-v) L_{n}(x)$ instead of $(x-u) L_{n}(x)$ we obtain

$$
\begin{aligned}
\text { (29) } & b_{n+1}(u-v) L_{n}(u)=\frac{((n+1) v-n w-1) L_{n+1}(w)+L_{n+2}(w)}{n+1}, \\
& \left(b_{n+1}-a_{n+2, n+1} b_{n+2}\right)(u-v) L_{n}(u) \\
(30)= & \frac{\left((n+1) v-n w+n^{2}+n-1\right) L_{n+1}(w)+((n+1) v-n(n+2)) L_{n+2}(w)}{n+1} .
\end{aligned}
$$

By considering the polynomial $\varphi(x)=x L_{n}(x)-(-1)^{n} x^{n+1} / n$ ! in applying Corollary 1 to $b^{T} A^{-2} \varphi(c)$ with $C(n),(4)$ and the use of Theorem 3 , we obtain

$$
b_{n+2}\left(a_{n+2, n+1} u L_{n}(u)-v L_{n}(v)\right)-b_{n+1} u L_{n}(u)=(n+1) L_{n+1}(w)-n L_{n}(w),
$$

and a similar application to $b^{T} c A^{-1} \varphi(c)$ with the condition (6) gives

$$
\begin{aligned}
& b_{n+2}(w-v)\left(a_{n+2, n+1} u L_{n}(u)-v L_{n}(v)\right)-b_{n+1}(w-u) u L_{n}(u) \\
& =(1-w) L_{n}(w)+(w-2) L_{n+1}(w)+L_{n+2}(w) .
\end{aligned}
$$

Theorem 14.

$$
v=\frac{n w+1}{n+1}+\frac{\left((w-1) L_{n}(w)+L_{n+1}(w)\right) L_{n+2}(w)}{n(n+1)\left(L_{n+1}(w)^{2}-L_{n}(w) L_{n+2}(w)\right)} .
$$

Proof. Multiplying (31) by $w-v$ and subtracting from (32) gives

$$
\begin{aligned}
b_{n+1}(u-v) u L_{n}(u) & =\frac{\left(n w-n^{2}-n+1\right) v-(n-1) w^{2}+\left(n^{2}-4\right) w+1}{n+1} L_{n+1}(w) \\
& +\frac{n(n+2) v-(n-1)(n+2) w-1}{n+1} L_{n+2}(w) .
\end{aligned}
$$

Eliminating $b_{n+1}$ from (29) and (33) yields

$$
\begin{aligned}
& v\left((n+1) L_{n+1}(w) u-\left(n w-n^{2}-n+1\right) L_{n+1}(w)-n(n+2) L_{n+2}(w)\right) \\
& =\left((n w+1) L_{n+1}(w)-L_{n+2}(w)\right) u-\left((n-1) w^{2}-\left(n^{2}-4\right) w-1\right) L_{n+1}(w) \\
& -((n-1)(n+2) w+1) L_{n+2}(w),
\end{aligned}
$$

and substituting for $u$ from Theorem 13 with the use of Theorem 3 gives the result. 
Once $w$ is chosen, $u$ and $v$ are determined by Theorems 13 and 14 . Equations (27) to (32) can then be used to determine $b_{n+1}, b_{n+2}$ and $a_{n+2, n+1}$. We consider a few possible choices of $w$ that satisfy the inequalities $w>x_{n}, w>u$ and $w>v$ so that all the abscissae will be contained in $[0,1]$ when scaled by the factor $1 / w$.

If $w$ is chosen as the largest zero of $L_{n+1}(x)$, then $u=n+1$ and $v=w-(w-1) / n$. This choice gives $w>x_{n}, w>u$ and $w>v$.

If $w$ is chosen as the largest zero of $L_{n+1}^{(1)}(x)$, then we have $L_{n+1}(w)=L_{n+2}(w)$ and it follows that $u=1$ and $v=w-(w-2) / n$. This choice also gives $w>x_{n}$, $w>u$ and $w>v$.

The choice of $w$ as a zero of $L_{n+2}^{(1)}(x)$ gives the damping condition $b^{T} A^{-2} e=0$ by Theorem 12 which implies $R(z)=O\left(z^{-2}\right)$ as $z \rightarrow \infty$. Theorems 13 and 14 give

$$
u=1+\frac{n w}{n+2}, \quad v=\frac{2(n+2)(n+3)+(n+3)\left(n^{2}+n-4\right) w-(n-2) w^{2}}{n((n+2)(n+3)-w)} .
$$

It can be verified numerically that if $w$ is chosen as one of the two largest zeros of $L_{n+2}^{(1)}(x)$, then it satisfies the inequalities $w>x_{n}, w>u$ and $w>v$.

The result $L_{n+3}(w)=0$ can be obtained by using $(I-A)^{n+3}=0$ and imposing the condition $b^{T} A^{2} c^{n}=w^{n+3} /((n+1)(n+2)(n+3))$ in addition to $C(n), B(n+1)$ and (5). It follows by evaluating $b^{T} A^{-1} e$ with $A^{-1}=\sum_{i=0}^{n+2}\left(\begin{array}{c}n+3 \\ i+1\end{array}\right)(-1)^{i} A^{i}$. Theorems 13 and 14 give

$$
\begin{aligned}
& u=\frac{n w-n^{2}-2 n+2}{n+2}, \\
& v=\frac{(n-2) w^{3}-(n+3)\left(2(n-3) w^{2}+\left(n^{3}+2 n^{2}+2 n+12\right) w+n^{2}+n-4\right)}{n\left(w^{2}-(n+3)\left(2 w+n^{2}+3 n+1\right)\right)} .
\end{aligned}
$$

In this case it can also be verified numerically that $w$ can be chosen as a zero of $L_{n+3}(x)$ satisfying the inequalities $w>x_{n}, w>u$ and $w>v$. For $n=2$ an interesting choice for $w$ is the third zero of $L_{5}(x)$. With this choice the method of order 4 in $y$ and order 3 in $z$ is $L$-stable.

\section{EXTENDED GENERALIZED SINGLY-IMPLICIT METHODS}

In this section we consider the construction of an $(n+m+1)$-stage extended generalized singly-implicit method by appending $m$ diagonally-implicit stages to the $n+1$ generalized singly-implicit stages and impose stiff accuracy for the cases $m=1,2,3$. We will always assume a stage order of $n+1,(I-A)^{n+m}=0$, $b^{T}=e_{n+m}^{T} A$ and impose $b^{T} A^{-2} c=1$ so that $\widetilde{R}(\infty)=0$.

6.1. One appended diagonally-implicit stage. For $m=1$, we have

$$
\widetilde{A}=\left[\begin{array}{ccccc}
0 & 0 & \cdots & 0 & 0 \\
a_{1,0} & a_{1,1} & \cdots & a_{1, n} & 0 \\
\vdots & \vdots & & \vdots & \vdots \\
a_{n, 0} & a_{n, 1} & \cdots & a_{n, n} & 0 \\
b_{0} & b_{1} & \cdots & b_{n} & 1
\end{array}\right]=\left[\begin{array}{cc}
0 & 0 \\
a_{0} & A
\end{array}\right],
$$




$$
\widetilde{b}=\left[\begin{array}{c}
b_{0} \\
b_{1} \\
\vdots \\
b_{n} \\
1
\end{array}\right]=\left[\begin{array}{c}
b_{0} \\
b
\end{array}\right], \quad \widetilde{c}=\left[\begin{array}{c}
0 \\
x_{1} \\
\vdots \\
x_{n} \\
w
\end{array}\right]=\left[\begin{array}{l}
0 \\
c
\end{array}\right] .
$$

As in Section 4, the positive abscissae $x_{1}<\cdots<x_{n}$ are the zeros of $L_{n}^{(1)}(x)$ and the $a_{i, j}, i, j=1, \ldots, n$, are determined by $C^{\star}(n+1)$, and $a_{i, 0}, i=1, \ldots, n$, by $C(1)$ in the same way. The weight vector $b$ is determined by $B^{\star}(n+1)$, and $b_{0}$ by $B(1)$.

Theorem 15. $\widetilde{R}(\infty)=L_{n+1}(w)$.

Proof. By $C^{\star}(n+1),(8)$ gives $(I-A) L_{k}(c)=L_{k+1}(c)-(A e-c)$ for $k \leq n$. Hence

$$
\begin{aligned}
L_{n+1}(c) & =(I-A)^{n+1} L_{0}(c)+\left(I+(I-A)+(I-A)^{2}+\cdots+(I-A)^{n}\right)(A e-c) \\
& =(I-A)^{n+1} e+\left(I-(I-A)^{n+1}\right)\left(e-A^{-1} c\right) \\
& =(I-A)^{n+1} A^{-1} c+e-A^{-1} c .
\end{aligned}
$$

Since $(I-A)^{n+1}=0$, it follows that $L_{n+1}(w)=b^{T} A^{-1} L_{n+1}(c)=1-b^{T} A^{-2} c=$ $\widetilde{R}(\infty)$.

The choice of $w$ as the largest zero of $L_{n+1}(x)$ gives a value greater than $x_{n}$ and a unique method of order $n+1$ in both $y$ and $z$ for index-2 DAEs with scaled abscissae contained in $[0,1]$.

6.2. Two appended diagonally-implicit stages. In this subsection we consider the case $m=2$ consisting of $n+1$ generalized singly-implicit stages with two appended diagonally-implicit stages,

$$
\widetilde{A}=\left[\begin{array}{cccccc}
0 & 0 & \cdots & 0 & 0 & 0 \\
a_{1,0} & a_{1,1} & \cdots & a_{1, n} & 0 & 0 \\
\vdots & \vdots & & \vdots & \vdots & \vdots \\
a_{n, 0} & a_{n, 1} & \cdots & a_{n, n} & 0 & 0 \\
a_{n+1,0} & a_{n+1,1} & \cdots & a_{n+1, n} & 1 & 0 \\
b_{0} & b_{1} & \cdots & b_{n} & b_{n+1} & 1
\end{array}\right], \quad \widetilde{b}=\left[\begin{array}{c}
b_{0} \\
b_{1} \\
\vdots \\
b_{n} \\
b_{n+1} \\
1
\end{array}\right], \quad \widetilde{c}=\left[\begin{array}{c}
0 \\
x_{1} \\
\vdots \\
x_{n} \\
v \\
w
\end{array}\right]
$$

Again we consider the partition given by (7). In addition to stiff accuracy, stage order $n+1$, and $(I-A)^{n+2}=0$, we will impose the conditions $B(n+2), b^{T} A^{-2} c=1$, and $b^{T} A^{-2} c^{n+2}=(n+2) w^{n+1}$ to obtain a unique method of order $n+2$ in both $y$ and $z$ for index-2 DAEs. It turns out to be possible to have all the scaled abscissae contained in the interval $[0,1]$. With stiff accuracy, stage order $n+1$ means $a_{0}=c-A e$ and $C^{\star}(n+1)$.

Theorem 16. $\widetilde{R}(\infty)=L_{n+2}(w)$.

Proof. As in the proof of Theorem 15, $C^{\star}(n+1)$ implies $L_{n+1}(c)=(I-A)^{n+1} A^{-1} c+$ $e-A^{-1} c$. By (8) with $\varphi(x)=L_{n+1}(x)-(-1)^{n+1} x^{n+1} /(n+1)$ !, we obtain

$$
\begin{aligned}
L_{n+2}(c) & =(I-A) L_{n+1}(c)+A e-c+\frac{(-1)^{n+2}}{(n+2) !}\left(c^{n+2}-(n+2) A c^{n+1}\right) \\
& =(I-A)^{n+2} A^{-1} c+e-A^{-1} c+\frac{(-1)^{n+2}}{(n+2) !}\left(c^{n+2}-(n+2) A c^{n+1}\right) .
\end{aligned}
$$


Since $(I-A)^{n+2}=0$ we have $L_{n+2}(w)=b^{T} A^{-1} L_{n+2}(c)=1-b^{T} A^{-2} c=\widetilde{R}(\infty)$ by $B^{\star}(n+2)$.

Now if $w$ is chosen as a zero of $L_{n+2}(x)$, we have an explicit formula for $v$ given by

\section{Theorem 17.}

$$
v=\frac{n w+n+2}{n+1} .
$$

Proof. By applying Corollary 1 with $\varphi(x)=x(x-v) L_{n}^{(1)}(x)-(-1)^{n} x^{n+2} / n !$ and the conditions $C^{\star}(n+1)$ and $b^{T} A^{-2} c^{n+2}=(n+2) w^{n+1}$, we obtain

$$
\begin{aligned}
b^{T} A^{-2}\left(c(c-v e) L_{n}^{(1)}(c)\right) & =\left(w(w-v) L_{n}^{(1)}(w)\right)^{\prime}, \\
w(w-v) L_{n}^{(1)}(w) & =w(w-v) L_{n}^{(1)^{\prime}}(w)+(2 w-v) L_{n}^{(1)}(w) .
\end{aligned}
$$

The result now follows by applying the identities in Theorem 3 with the use of $L_{n+2}(w)=0$.

As a corollary, $w-v=(w-n-2) /(n+1)=(v-n-2) / n$. Now the zeros of $L_{n+2}(x)$ have sum $(n+2)^{2}$ and average $n+2$. Hence if $w$ is the largest zero, then $w>n+2$ and it follows that $w>v>n+2$. By the interlacing property of Laguerre polynomials, $w$ is greater than the largest zero of $L_{n+1}(x)$ which, in turn, is greater than the largest zero of $L_{n+1}^{\prime}(x)$. Since $L_{n}^{(1)}(x)=-L_{n+1}^{\prime}(x)$, we have that $w$ is greater than the largest zero of $L_{n}^{(1)}(x)$ and hence that all the abscissae scaled by the factor $1 / w$ will be contained in the interval $[0,1]$.

The coefficient $b_{n+1}$ is determined from the relation $(n+2) b_{n+1} v L_{n}^{(1)}(v)=$ $-(n+1) w L_{n+1}^{(1)}(w)$, obtained by applying (9) with the use of $B^{\star}(n+2), \varphi(x)=$ $x L_{n}^{(1)}(x)$ and the identities in Theorems 3 and 4 .

6.3. Three appended diagonally-implicit stages. We now consider the case $m=3$ consisting of an $(n+1)$-stage generalized singly-implicit block with three appended diagonally-implicit stages,

$$
\begin{gathered}
\widetilde{A}=\left[\begin{array}{ccccccc}
0 & 0 & \cdots & 0 & 0 & 0 & 0 \\
a_{1,0} & a_{1,1} & \cdots & a_{1, n} & 0 & 0 & 0 \\
\vdots & \vdots & & \vdots & \vdots & \vdots & \vdots \\
a_{n, 0} & a_{n, 1} & \cdots & a_{n, n} & 0 & 0 & 0 \\
a_{n+1,0} & a_{n+1,1} & \cdots & a_{n+1, n} & 1 & 0 & 0 \\
a_{n+2,0} & a_{n+2,1} & \cdots & a_{n+2, n} & a_{n+2, n+1} & 1 & 0 \\
b_{0} & b_{1} & \cdots & b_{n} & b_{n+1} & b_{n+2} & 1
\end{array}\right] \\
\widetilde{b}=\left[\begin{array}{c}
b_{0} \\
b_{1} \\
\vdots \\
b_{n} \\
b_{n+1} \\
b_{n+2} \\
1
\end{array}\right], \quad \widetilde{c}=\left[\begin{array}{c}
0 \\
x_{1} \\
\vdots \\
x_{n} \\
u \\
v \\
w
\end{array}\right],
\end{gathered}
$$


and partitioned according to (7). In addition to stiff accuracy, stage order $n+1$ and $(I-A)^{n+3}=0$, we will impose $B(n+3)$ and some additional conditions to obtain order $n+3$ in $y$ and order $n+2$ in $z$ in terms of $w=\widetilde{b}^{T} \widetilde{e}$. These include a condition for order $n+2$ in $z$ given by (4) and conditions for order $n+3$ in $y$ given by (5) and (6).

Theorem 18. $\widetilde{R}(\infty)=L_{n+3}(w)$.

Proof. By $C^{\star}(n+1)$, application of (8) with

$$
\varphi(x)=L_{n+2}(x)-\frac{(-1)^{n+2}}{(n+2) !} x^{n+2}-\frac{(-1)^{n+1}}{(n+1) !}(n+2) x^{n+1}
$$

yields

$$
\begin{aligned}
L_{n+3}(c)= & (I-A) L_{n+2}(c)+A e-c+\frac{(-1)^{n+3}}{(n+3) !}\left(c^{n+3}-(n+3) A c^{n+2}\right) \\
& -\frac{(-1)^{n+1}}{(n+1) !}\left(c^{n+2}-(n+2) A c^{n+1}\right) .
\end{aligned}
$$

Substituting for $L_{n+2}(c)$ from the proof of Theorem 16, the result follows from evaluating $L_{n+3}(w)=b^{T} A^{-1} L_{n+3}(c)$ with the use of $(I-A)^{n+3}=0$, and the conditions $B^{\star}(n+3)$ and $(5)$.

With $w$ chosen as a zero of $L_{n+3}(x)$ greater than the largest zero of $L_{n}^{(1)}(x)$, we now consider the determination of $u, v, b_{n+1}, b_{n+2}$ and $a_{n+2, n+1}$.

We first present some results for ease of reference in the proofs of the two main results that follow. They all assume that $L_{n+3}(w)=0$ and can be verified with the use of Theorems 3 and 4.

$$
\begin{aligned}
w L_{n}^{(1)}(w) & =\frac{w^{2}-(3 n+7) w+(n+2)(n+3)}{n+2} L_{n+2}(w), \\
\left(w L_{n}^{(1)}(w)\right)^{\prime} & =\frac{w^{2}-(4 n+8) w+3 n^{2}+12 n+11}{n+2} L_{n+2}(w), \\
\int_{0}^{w} x L_{n}^{(1)}(x) d x & =\frac{w^{2}-(2 n+6) w+n+3}{n+2} L_{n+2}(w), \\
\int_{0}^{w} x^{2} L_{n}^{(1)}(x) d x & =\frac{w^{3}-(2 n+7) w^{2}+(2 n+8) w-2}{n+2} L_{n+2}(w) .
\end{aligned}
$$

By $B^{\star}(n+3)$, application of $(9)$ with $\varphi(x)=x(x-u) L_{n}^{(1)}(x)$ yields

$$
\begin{aligned}
b_{n+2} v(v-u) L_{n}^{(1)}(v)+w(w-u) L_{n}^{(1)}(w) & =b^{T} c(c-u e) L_{n}^{(1)}(c) \\
& =\int_{0}^{w} x(x-u) L_{n}^{(1)}(x) d x .
\end{aligned}
$$

Similarly, applying Corollary 1 with $\varphi(x)=x(x-u) L_{n}^{(1)}(x)-(-1)^{n} x^{n+2} / n$ ! and the conditions $C^{\star}(n+1)$ and $(4)$ gives

$$
\begin{aligned}
-b_{n+2} v(v-u) L_{n}^{(1)}(v)+w(w-u) L_{n}^{(1)}(w) & =b^{T} A^{-2} c(c-u e) L_{n}^{(1)}(c) \\
& =(w-u)\left(w L_{n}^{(1)}(w)\right)^{\prime}+w L_{n}^{(1)}(w) .
\end{aligned}
$$

Theorem 19.

$$
u=\frac{n w+n+4}{n+2} .
$$


Proof. By adding (38) and (39) we obtain an explicit expression for $u$,

$$
u=\frac{w\left(w L_{n}^{(1)}(w)\right)^{\prime}+(1-2 w) w L_{n}^{(1)}(w)+\int_{0}^{w} x^{2} L_{n}^{(1)}(x) d x}{\left(w L_{n}^{(1)}(w)\right)^{\prime}-2 w L_{n}^{(1)}(w)+\int_{0}^{w} x L_{n}^{(1)}(x) d x},
$$

and the result follows by use of the formulae (34) to (37).

Applying Corollary 1 with $\varphi(x)=x^{2} L_{n}^{(1)}(x)-(-1)^{n} x^{n+2} / n$ ! and the use of the condition (4) yields

$$
\begin{aligned}
& -b_{n+1} u^{2} L_{n}^{(1)}(u)+b_{n+2}\left(a_{n+2, n+1} u^{2} L_{n}^{(1)}(u)-v^{2} L_{n}^{(1)}(v)\right)+w^{2} L_{n}^{(1)}(w) \\
& =b^{T} A^{-2} c^{2} L_{n}^{(1)}(c)=\left(w^{2} L_{n}^{(1)}(w)\right)^{\prime},
\end{aligned}
$$

while the use of the condition (6) gives

$$
\begin{aligned}
& b_{n+1}(u-w) u^{2} L_{n}^{(1)}(u)+b_{n+2}(w-v)\left(a_{n+2, n+1} u^{2} L_{n}^{(1)}(u)-v^{2} L_{n}^{(1)}(v)\right)+w^{3} L_{n}^{(1)}(w) \\
& =b^{T} c A^{-1} c^{2} L_{n}^{(1)}(c)=w^{3} L_{n}^{(1)}(w)-\int_{0}^{w} x^{2} L_{n}^{(1)}(x) d x .
\end{aligned}
$$

Multiplying (40) by $w-v$ and subtracting (41) from the result gives

$$
\begin{aligned}
b_{n+1}(v-u) u^{2} L_{n}^{(1)}(u)+(w-v) w^{2} L_{n}^{(1)}(w) \\
\quad=(w-v)\left(w^{2} L_{n}^{(1)}(w)\right)^{\prime}+\int_{0}^{w} x^{2} L_{n}^{(1)}(x) d x .
\end{aligned}
$$

Furthermore, applying (9) with $\varphi(x)=x(x-v) L_{n}^{(1)}(x)$ and the use of the conditions $B^{\star}(n+3)$ and (6) yields

$$
\begin{aligned}
b_{n+1}(u-v) u L_{n}^{(1)}(u)+w(w-v) L_{n}^{(1)}(w) & =b^{T} c(c-v e) L_{n}^{(1)}(c) \\
& =\int_{0}^{w} x(x-v) L_{n}^{(1)}(x) d x .
\end{aligned}
$$

Theorem 20.

$$
v=\frac{(n-2) w^{3}-\left(n^{3}+4 n^{2}+n-18\right) w^{2}-2\left(n^{2}+8 n+18\right) w+4(n+3)}{n\left(w^{2}-\left(n^{2}+5 n+8\right) w+n+3\right)} .
$$

Proof. Multiplying (43) by $u$ and adding to (42) gives an explicit expression for $v$ in terms of $u$ and $w$ in the form $v=P / Q$, where

$$
\begin{aligned}
P=u & \left(\int_{0}^{w} x^{2} L_{n}^{(1)}(x) d x-w^{2} L_{n}^{(1)}(w)\right)+(1-w) w^{2} L_{n}^{(1)}(w)+w^{2}\left(w L_{n}^{(1)}(w)\right)^{\prime} \\
& +\int_{0}^{w} x^{2} L_{n}^{(1)}(x) d x \\
Q=u & \left(\int_{0}^{w} x L_{n}^{(1)}(x) d x-w L_{n}^{(1)}(w)\right)+(1-w) w L_{n}^{(1)}(w)+w\left(w L_{n}^{(1)}(w)\right)^{\prime} .
\end{aligned}
$$

Applying (34) to (37) now gives $v=N / D$, where

$$
\begin{aligned}
& \begin{aligned}
& N=u\left(n w^{2}-\left(n^{2}+3 n-2\right) w-2\right)-(n-1) w^{3}+\left(2 n^{2}+2 n-9\right) w^{2} \\
& \quad \quad\left(n^{2}+7 n+14\right) w-2, \\
& D=u(n+1)(w-n-3)-n w^{2}+\left(2 n^{2}+4 n-2\right) w+(n+2)(n+3) .
\end{aligned}
\end{aligned}
$$

Substituting for $u$ from Theorem 19 then yields the result for $v$ in terms of $w$. 
With $w$ chosen as a zero of $L_{n+3}(x)$, and $x_{n}$ denoting the largest zero of $L_{n}^{(1)}(x)$, $u$ and $v$ are respectively determined by Theorems 19 and 20. With the use of (34) to (37), the coefficient $b_{n+2}$ can be determined by (38) and (39), $b_{n+1}$ by (42) and (43), and $a_{n+2, n+1}$ by (40) and (41). If $w$ is the largest zero of $L_{n+3}(x)$, then it is clear that $w>x_{n}$ and $w>u$. Numerical computations also show that $w>v$. Hence all the scaled abscissae will be contained in the interval $[0,1]$ for this choice of $w$. It can also be verified numerically that these inequalities hold for $1 \leq n \leq 10$ if $w$ is the second largest zero of $L_{n+3}(x)$.

\section{TRANSFORMATIONS}

For the methods derived in Section 5 (respectively Section 6), explicit fomulas have been given for the elements in columns $n+1, n+2, \ldots, n+m$ (respectively $n+2, n+3, \ldots, n+m+1)$ of the $A$ matrix. In this section we derive formulas for the remaining elements making use of similarity transformations. Although implementation details will not be discussed it will be shown in a later paper that, as for standard singly-implicit methods, these transformations play a central role in the practical implementation of the methods.

7.1. Transformations for the extended methods. For the methods of Section 5, define the matrix $V$ by

$$
V=\left[L_{0}(c), L_{1}(c), \ldots, L_{n-1}(c), e_{n+1}, e_{n+2}, \ldots, e_{n+m}\right] .
$$

Theorem 21. For the methods of Section 5, A is given by

$$
A=V \bar{A} V^{-1},
$$

where

$$
A=\left[\begin{array}{cc}
A_{11} & 0 \\
A_{21} & A_{22}
\end{array}\right], \quad \bar{A}=\left[\begin{array}{cc}
\bar{A}_{11} & 0 \\
\bar{A}_{21} & A_{22}
\end{array}\right]
$$

with

$$
\bar{A}_{11}=\left[\begin{array}{cccccc}
1 & 0 & 0 & \cdots & 0 & 0 \\
-1 & 1 & 0 & \cdots & 0 & 0 \\
0 & -1 & 1 & \cdots & 0 & 0 \\
\vdots & \vdots & \vdots & & \vdots & \vdots \\
0 & 0 & 0 & \cdots & -1 & 1
\end{array}\right], \quad \bar{A}_{21}=\left[\begin{array}{cccccc}
0 & 0 & 0 & \cdots & 0 & -L_{n}\left(x_{n+1}\right) \\
0 & 0 & 0 & \cdots & 0 & -L_{n}\left(x_{n+2}\right) \\
0 & 0 & 0 & \cdots & 0 & -L_{n}\left(x_{n+3}\right) \\
\vdots & \vdots & \vdots & & \vdots & \vdots \\
0 & 0 & 0 & \cdots & 0 & -L_{n}\left(x_{n+m}\right)
\end{array}\right]
$$

Proof. We will prove the equivalent result that $A V=V \bar{A}$. By the conditions $C(n)$, column $i$ of $A V(i \leq n)$ is given by $\int_{0}^{c} L_{i-1}(x) d x=L_{i-1}(c)-L_{i}(c)$ which equals the corresponding column of $V \bar{A}$. The remaining columns of $A V$ and $V \bar{A}$ are each the same as for $A$.

To simplify the computation of the elements of $A$, the next result gives explicit formulas for the elements of $V^{-1}$. 
Theorem 22. The $(i, j)$-th element of $V^{-1}$ is given by

$$
\begin{cases}\frac{x_{j} L_{i-1}\left(x_{j}\right)}{n^{2} L_{n-1}\left(x_{j}\right)^{2}}, & i \leq n, j \leq n, \\ 0, & i \leq n, j>n, \\ \frac{x_{j} L_{n}\left(x_{i}\right)}{n\left(x_{i}-x_{j}\right) L_{n-1}\left(x_{j}\right)}, & i>n, j \leq n, \\ \delta_{i j}, & i>n, j>n .\end{cases}
$$

Proof. Write the matrix $V$ in partitioned form

$$
V=\left[\begin{array}{ll}
V_{11} & 0 \\
V_{21} & I
\end{array}\right]
$$

where $V_{11}$ is $n \times n$. The inverse in partitioned form is

$$
V^{-1}=\left[\begin{array}{cc}
V_{11}^{-1} & 0 \\
-V_{21} V_{11}^{-1} & I
\end{array}\right]
$$

The elements in the $(1,1)$ block are given by Corollary 3 and the $(1,2)$ and $(2,2)$ blocks are trivial. It remains to prove that for $i>n, j \leq n$, the $(i-n, j)$-th element of $-V_{21} V_{11}^{-1}$ is as claimed. To evaluate this element, use (23) to give the result

$$
\begin{aligned}
-\sum_{k=1}^{n} L_{k-1} & \left(x_{i}\right) \frac{L_{k-1}\left(x_{j}\right) x_{j}}{n^{2} L_{n-1}\left(x_{j}\right)^{2}}=-\frac{x_{j}}{n^{2} L_{n-1}\left(x_{j}\right)^{2}} \sum_{k=1}^{n} L_{k-1}\left(x_{i}\right) L_{k-1}\left(x_{j}\right) \\
= & -\frac{x_{j}}{n^{2} L_{n-1}\left(x_{j}\right)^{2}} \frac{n}{x_{i}-x_{j}}\left(L_{n}\left(x_{j}\right) L_{n-1}\left(x_{i}\right)-L_{n}\left(x_{i}\right) L_{n-1}\left(x_{j}\right)\right) \\
= & \frac{x_{j} L_{n}\left(x_{i}\right)}{\left(x_{i}-x_{j}\right) n L_{n-1}\left(x_{j}\right)},
\end{aligned}
$$

where we note that $L_{n}\left(x_{j}\right)=0$.

7.2. Transformations for the extended generalized methods. For the methods of Section 6 , we will carry out a similar analysis. First we deal with the vector $a_{0}$ defined by $c-A e$.

Theorem 23. The elements of $a_{0}$ are given by

$$
a_{i, 0}= \begin{cases}\frac{1}{n+1}\left(1+L_{n-1}^{(1)}\left(x_{i}\right)\right), & i \leq n, \\ \frac{1}{n+1}\left(1+L_{n-1}^{(1)}\left(x_{i}\right)+\left(\frac{x_{i}}{n+1}-1\right) L_{n}^{(1)}\left(x_{i}\right)-\sum_{j>i} a_{i j} L_{n}^{(1)}\left(x_{j}\right)\right), & i>n .\end{cases}
$$

Proof. By $C^{\star}(n+1)$, application of $(8)$ with $\varphi(x)=L_{n}^{(1)}(x)$ gives

$$
(n+1) a_{0}=\int_{0}^{c} L_{n}^{(1)}(x) d x-A L_{n}^{(1)}(c),
$$

and the result follows by use of the various identities in Theorems 3 and 4 .

To evaluate the first $n$ columns of $A$, form the tranformation matrix $V$ given by

$$
V=\left[c L_{0}^{(1)}(c), \frac{1}{2} c L_{1}^{(1)}(c), \ldots, \frac{1}{n} c L_{n-1}^{(1)}(c), e_{n+1}, e_{n+2}, \ldots, e_{n+m}\right] \text {. }
$$

We then have 
Theorem 24. For the methods of Section 6, $A$ is given by

$$
A=V \bar{A} V^{-1},
$$

where

$$
A=\left[\begin{array}{cc}
A_{11} & 0 \\
A_{21} & A_{22}
\end{array}\right], \quad \bar{A}=\left[\begin{array}{cc}
\bar{A}_{11} & 0 \\
\bar{A}_{21} & A_{22}
\end{array}\right],
$$

with

$$
\begin{gathered}
\bar{A}_{11}=\left[\begin{array}{cccccc}
1 & 0 & 0 & \cdots & 0 & 0 \\
-1 & 1 & 0 & \cdots & 0 & 0 \\
0 & -1 & 1 & \cdots & 0 & 0 \\
\vdots & \vdots & \vdots & & \vdots & \vdots \\
0 & 0 & 0 & \cdots & -1 & 1
\end{array}\right], \\
\bar{A}_{21}=\left[\begin{array}{cccccc}
0 & 0 & 0 & \cdots & 0 & -\frac{x_{n+1}}{n+1} L_{n}^{(1)}\left(x_{n+1}\right) \\
0 & 0 & 0 & \cdots & 0 & -\frac{x_{n+2}}{n+1} L_{n}^{(1)}\left(x_{n+2}\right) \\
0 & 0 & 0 & \cdots & 0 & -\frac{x_{n+3}}{n+1} L_{n}^{(1)}\left(x_{n+3}\right) \\
\vdots & \vdots & \vdots & & \vdots & \vdots \\
0 & 0 & 0 & \cdots & 0 & -\frac{x_{n+m}}{n+1} L_{n}^{(1)}\left(x_{n+m}\right)
\end{array}\right] .
\end{gathered}
$$

Proof. This is proved in a similar way to Theorem 21.

We now give explicit formulas for the elements of the inverse of the new transformation matrix.

Theorem 25. The $(i, j)$-th element of $V^{-1}$ is given by

$$
\begin{cases}\frac{L_{i-1}^{(1)}\left(x_{j}\right)}{(n+1) L_{n-1}^{(1)}\left(x_{j}\right)^{2}}, & i \leq n, j \leq n, \\ 0, & i \leq n, j>n, \\ \frac{x_{i} L_{n}^{(1)}\left(x_{i}\right)}{\left(x_{i}-x_{j}\right)(n+1) L_{n-1}^{(1)}\left(x_{j}\right)}, & i>n, j \leq n, \\ \delta_{i j}, & i>n, j>n .\end{cases}
$$

Proof. This is proved as for Theorem 22.

\section{CONCLUDing REMARKS}

This paper has concentrated on the derivation of a large range of possible methods which we believe will be effective for the solution of index-2 DAEs. If we required only a single method, without error estimation capabilities, then the method presented in Subsection 6.3 would be particularly attractive. Although error estimation and other implementation issues could not be included in this paper, it is intended to discuss them in a later publication. At the same time, results of numerical tests will be presented.

\section{ACKNOWLEDGEMENTS}

The authors wish to acknowledge valuable discussions with Roger Alexander and Philippe Chartier. 


\section{REFERENCES}

[1] M. Abramovitch and I. A. Stegun, Handbook of Mathematical Functions, with Formulas, Graphs and Mathematical Tables, Dover Publications, New York (1966). MR 34:8606

[2] K. Burrage, J. C. Butcher and F.H. Chipman, An implementation of singly-implicit RungeKutta methods, BIT 20 (1980), 326-340. MR 82h:65049

[3] K. Burrage and R.P.K. Chan, On smoothing and order reduction effects for implicit RungeKutta formulae, J. Comput. Appl. Math. 45 (1993), 17-27. MR 93j:65110

[4] J.C. Butcher, The Numerical Analysis of Ordinary Differential Equations: Runge-Kutta and General Linear Methods, J. Wiley and Sons, Chichester and New York (1987). MR 88d:65002

[5] J. C. Butcher, Towards efficient implementation of singly-implicit methods, ACM Trans. Math. Software 14 (1988), 68-75. MR 89b:65167

[6] J. C. Butcher and J.R. Cash, Towards efficient Runge-Kutta methods for stiff systems, SIAM J. Numer. Anal. 27 (1990), 753-761. MR 91a:65171

[7] R.P.K. Chan and P. Chartier, Gaussian symmetrizers for index-2 differential algebraic equations (unpublished notes, 1994).

[8] A. Erdélyi, W. Magnus, F. Oberhettinger and F.G. Tricomi, Higher Transcendental Functions, volume II, Bateman Manuscript Project, McGraw-Hill, New York, Toronto, London (1953). MR 15:419i

[9] E. Hairer, Ch. Lubich and M. Roche, The Numerical Solution of Differential Algebraic Systems by Runge-Kutta Methods. Lecture Notes in Math. 1409, Springer Verlag (1989). MR 91a:65178

Department of Mathematics, The University of Auckland, Auckland, New Zealand E-mail address: butcher@math.auckland.ac.nz

Division of Science and Technology, Tamaki Campus, The University of Auckland, Auckland, New Zealand

E-mail address: chan@scitec.auckland.ac.nz 